\title{
Coulomb interactions and effective quantum inertia of charge carriers in a macroscopic conductor
}

\author{
A. Delgard, ${ }^{1}$ B. Chenaud, ${ }^{1}$ U. Gennser, ${ }^{2}$ A. Cavanna, ${ }^{2}$ D. Mailly, ${ }^{2}$ P. Degiovanni $\odot,{ }^{3}$ and C. Chaubet ${ }^{1, *}$ \\ ${ }^{1}$ Université Montpellier 2, CNRS, Laboratoire Charles Coulomb UMR5221, F-34095 Montpellier, France \\ ${ }^{2}$ Centre de Nanosciences et de Nanotechnologies (C2N), CNRS, Université Paris-Sud, Université Paris-Saclay, F-91120 Palaiseau, France \\ ${ }^{3}$ Université Lyon, Ens de Lyon, Université Claude Bernard Lyon 1, CNRS, Laboratoire de Physique, F-69342 Lyon, France
}

(Received 24 August 2020; accepted 3 August 2021; published 29 September 2021)

\begin{abstract}
We study the low-frequency admittance of a quantum Hall bar of a size much larger than the electronic coherence length. We find that this macroscopic conductor behaves as an ideal quantum conductor with vanishing longitudinal resistance and purely inductive behavior up to $f \lesssim 1 \mathrm{MHz}$. Using several measurement configurations, we study the dependence of this inductance on the length of the edge channel and on the integer quantum Hall filling factor. The experimental data are well described by a scattering model for edge magnetoplasmons taking into account the effective long range Coulomb interactions within the sample. We find that the inductance's dependence on the filling factor arises predominantly from the effective quantum inertia of charge carriers induced by Coulomb interactions.
\end{abstract}

DOI: 10.1103/PhysRevB.104.L121301

By demonstrating that macroscopic conductors could exhibit robust dc transport properties of quantum origin, the integer quantum Hall effect (IQHE) [1-5] has been a major surprise. The importance of this breakthrough for metrology was acknowledged immediately [1] and has led to the redefinition of the ohm [6]. The finite frequency response of quantum Hall conductors has been intensively studied by metrologists: The use of an ac bridge at finite frequency $f$ revealed a departure of the Hall resistance $R_{H}(f)$ at $v=2$ from the expected value $R_{K} / 2=h / 2 e^{2}$ [7-11]. It was then attributed to intrinsic inductances and capacitances $[12,13]$. Later, Schurr et al. proposed a double shielded sample allowing for a frequency-independent resistance standard [14], but these works left open the question of the origin of these capacitances and inductances.

On the other hand, the finite frequency transport properties of quantum coherent conductors, of a size smaller than the electron coherence length, are expected to be dominated by quantum effects. For low-dimensional conductors such as carbon nanotubes [15], or graphene [16], the inductance is of a purely kinetic origin. Small superconducting inductors $[17,18]$ now used in space industry [19] are based on the inertia of Cooper pairs. For a quantum coherent conductor, the theory developed by Büttiker and his collaborators [20-22] relates the associated $L / R$ or $R C$ times to the Wigner-Smith time delay for charge carriers scattering across the conductor. These remarkable predictions have been confirmed by the measurement of the finite frequency admittance of quantum Hall $R-C$ [23] and $R-L[24,25]$ circuits of $\mu \mathrm{m}$ size in the $\mathrm{GHz}$ range at cryogenic temperatures.

In this Letter, we demonstrate that, in the ac regime, a mm long ungated macroscopic quantum Hall bar, of a size much larger than the electronic coherence length, exhibits a finite inductance as well as a vanishing longitudinal resistance. Such

*Corresponding author: christophe.chaubet@umontpellier.fr a purely inductive longitudinal response is expected for quantum conductors with zero backscattering: A kinetic energy cost proportional to the square of the current arises from both the Pauli principle and the linear dispersion relation for electrons close to the Fermi level (see Sec. I of the Supplemental Material [26]). This effective inertia of carriers causes the current response to lag the applied electric field. Here, we identify an inductance of the order of tens of $\mu \mathrm{H} \mathrm{mm}^{-1}$ and connect it to an effective velocity $v_{\text {eff }}$ along the quantum Hall bar's edges. Contrary to gated samples, in which $v_{\text {eff }}$ is almost independent of the filling factor $v$ [24], we show that, because of the Coulomb interactions between opposite edges of the sample, $v_{\text {eff }}$ depends on $v$ in our samples. Using the edge-magnetoplasmon scattering approach combined with a discrete element approach in the manner of Büttiker, we show that

$$
\frac{v_{\mathrm{eff}}(v)}{v_{\mathrm{d}}(v)}=1+\frac{v \alpha_{\mathrm{eff}}(v)}{\pi} \ln \left[\frac{W / \xi_{H}(v)}{v}\right]
$$

for a sample of width $W$. Here, $v_{\mathrm{d}}(v)$ represents the charge density wave velocity along the system of $v$-copropagating chiral edge channels, neglecting Coulomb interactions with the other (counterpropagating) edge channels. In a Büttiker view of the edge channels [27] as well as in models assuming that no compressible stripes are present at the edge [28,29], $v_{\mathrm{d}}(v)$ is the drift velocity of noninteracting electrons at the edge in an effective confining potential $U_{v}$ and, therefore, it plays the role of an effective Fermi velocity in the onedimensional (1D) linear dispersion relation along the edge [30]. In the presence of compressible stripes, which appear for a sufficiently smooth confining potential [31], it corresponds to the effective velocity of the charge density mode in the system of $v$-copropagating edge channels [32], taking into account the presence of the incompressible part of the edge channel [33]. Nevertheless, we denote it by $v_{\mathrm{d}}(\nu)$ because, in a model of an edge channel without compressible parts, it really would be an electronic drift velocity. Importantly, 

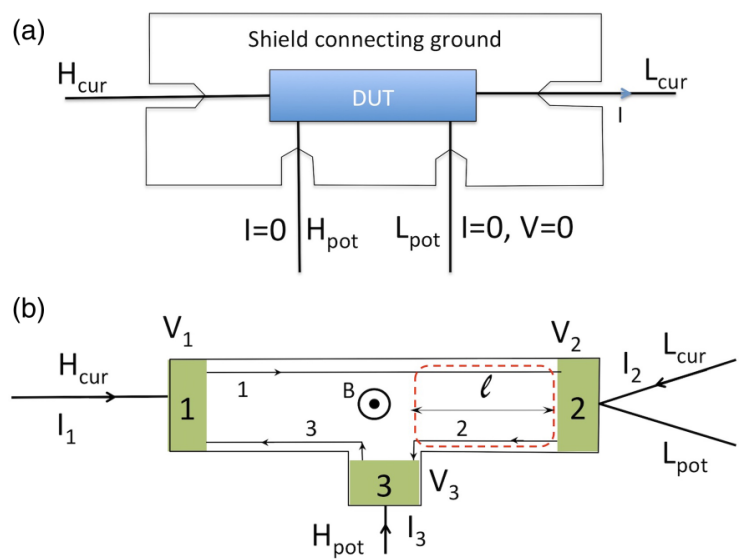

FIG. 1. (a) The device under test (DUT) is measured using four coaxial cables and the impedance-meter Agilent HP4294A, which measures the current $I$ at $L_{\text {cur }}$, the potential $V$ at $H_{\text {pot }}$, and gives $G=I / V$ (for details, see Refs. [36,37]). Note that the potentials of $H_{\text {pot }}, L_{\text {pot }}, H_{\text {cur }}$, and $L_{\text {cur }}$ are the potentials of the four connectors of the impedance meter. (b) Scheme of the multiterminal Hall bar with only three ohmic contacts wire-bonded onto the sample holder. In this geometry, the impedance is $Z_{23}^{\text {(expt) }}(\omega)=-\left(\partial V_{3} / \partial I_{2}\right)(\omega)$ for $V_{2}=0$.

this velocity deviates from the classical $1 / B$ behavior of the electronic drift velocity because of screening effects, which change the electrostatic potential at the edge as $v$ varies.

Here, $\alpha_{\text {eff }}(v)$ denotes the effective fine structure constant $\left(\alpha_{\text {qed }}\right.$ in the vacuum) at filling factor $\nu$ : $\alpha_{\text {eff }}(v)=$ $\left(\alpha_{\text {qed }} / \varepsilon_{r}\right)\left[c / v_{\mathrm{d}}(v)\right]$. The length $\xi_{H}(v)$, which also depends on $\nu$, is an effective renormalized width of a single edge channel of the order of the width of incompressible edge channels $\lambda_{H}(v)$ [31] (see Sec. IV of the Supplemental Material [26]).

Our work demonstrates that the purely inductive response of the macroscopic ungated quantum Hall bar reflects the effective quantum inertia of charge carriers renormalized by Coulomb interactions within the sample. Therefore, although electron transport across such a conductor is not coherent, its $\mathrm{dc}$ and ac transport properties are of quantum origin, a fact that ultimately relies on the coherence of edge-magnetoplasmon (EMP) modes propagating along chiral edge channels. EMP coherence has enabled the demonstration of single and double EMP Fabry-Pérot interferometers [34] as well as of a MachZehnder plasmonic interferometer [35].

Using shallow etching, our samples are processed on an $\mathrm{AlGaAs} / \mathrm{GaAs}$ heterojunction with the two-dimensional electron gas (2DEG) located at the heterointerface $(105 \mathrm{~nm}$ beneath the surface) with carrier density $n_{s}=5.1 \times 10^{11} \mathrm{~cm}^{-2}$ and mobility $\mu=30 \mathrm{~m}^{2} / \mathrm{Vs}$. We have processed a $2 \times$ $0.4 \mathrm{~mm}^{2}$ ungated Hall bar which exhibits a sufficiently large kinetic inductance. The sample has no back gate and is glued on a ceramic sample holder to avoid parasitic capacitances. It is placed at the center of a high magnetic field at $1.5 \mathrm{~K}$.

In the measurement setup depicted in Fig. 1(a), the current is injected using $H_{\text {cur }}$ ( $5 \mathrm{mV}$ bias), and measured using $L_{\text {cur }}$. The potential of $H_{\text {pot }}$ is measured while $V=0$ and $I=0$ are imposed at $L_{\text {pot }}$. The current intensity $(\lesssim 0.5 \mu \mathrm{A}$ at $v=2)$ remains below the breakdown current and currents used in metrology [4,7]. For each value of $B$, the resistance and the

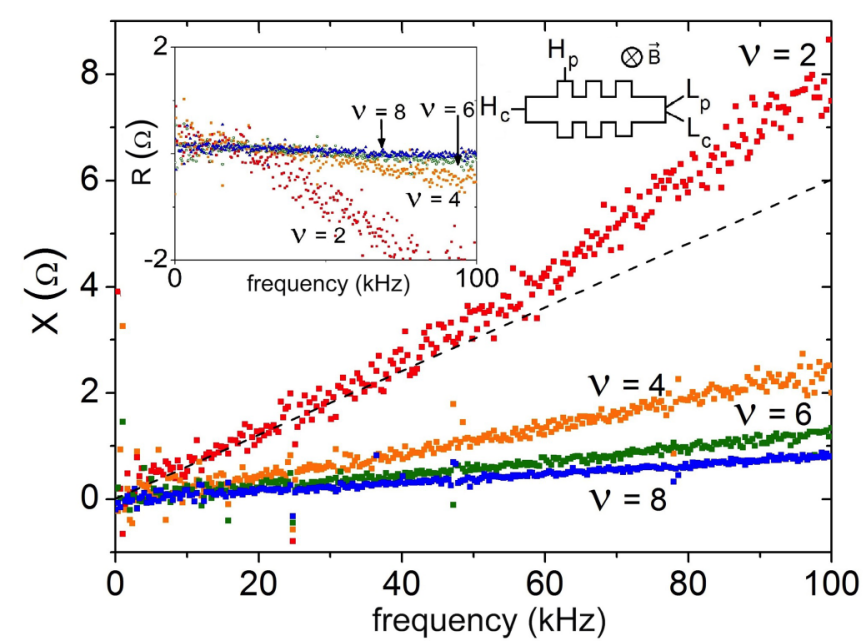

FIG. 2. The reactance $X$ as a function of the frequency $f$ for different $v$ and $B_{<0}$, in the measurement configuration shown here. Inset: The longitudinal resistance $R(f)$ vanishes quadratically for integer filling factors at low frequency.

reactance have been measured for 300 values of the frequency $f$ in the range $40 \mathrm{~Hz}-100 \mathrm{kHz}$.

Due to chirality of the quantum Hall transport, an ohmic contact wire-bonded to the sample holder and so to a coaxial cable, generates a leakage current through the cable capacitance if the potential does not vanish $[38,39]$. This results in a faulty measurement $[40,41]$. For this reason, all results presented here have been carried out at integer filling factors, where the longitudinal resistance $R_{x x}(\omega)$ vanishes [42]. Furthermore, only three of the ohmic contacts processed on the sample were wire-bonded onto the sample holder as shown in Fig. 1(b). To measure a zero resistance state, the third contact is inserted along the edge connected to the reference potential. In $\mathrm{dc}$, one would measure a potential $V_{H_{\mathrm{pot}}}=0$. In ac, $V_{H_{\mathrm{pot}}} \neq 0$ and we measure the frequency-dependent impedance $Z_{23}^{(\text {expt })}(\omega)=-\partial V_{3} /\left.\partial I_{2}\right|_{V_{2}=0}(\omega)$. Different configurations and edge channel lengths can be obtained by recabling the contacts and changing the sample side (in this case, the magnetic field orientation must be reversed). We have also wire-bonded a fourth ohmic contact on the same side of the sample to connect $L_{\text {pot }}$ to access another edge channel length.

Figure 2 presents unfiltered and nonaveraged raw data for the reactance $X(f)=\operatorname{Im}\left[Z_{23}^{(\text {expt }}(2 \pi f)\right]$ in a given sample configuration for $v=2,4,6$, and 8 . The positive linear dependence of $X(f)$ is the signature of an inductive behavior. The corresponding inductance decreases with $v$. These data are completely reproducible in the regions of magnetic fields where $R_{x x}=0$. This is a key point of our work: For integer filling factors, the real part $R(f)=\operatorname{Re}\left[Z_{23}^{(\text {expt })}(2 \pi f)\right]$ of the measured impedance is close to zero with values between $\pm 0.5 \Omega$ at low frequency as shown in the inset of Fig. 2 . These results extend the work of Gabelli et al. [24] in which the sample resistance was $R_{H}=R_{K} / v$, to the case of a zero resistance macroscopic device. At higher frequencies, a small real part of the measured response function $R(f)$ appears. This effect is discussed in Sec. II of the Supplemental Material [26] and is related to the deviation of the reactance $X(f)$ from linearity seen in Fig. 2. 
Since the ac transport properties of a quantum Hall conductor are directly related to the scattering of edge magnetoplasmons [43-46], already used to study EMP propagation [47,48], charge fractionalization [49-52], and electronic relaxation and decoherence [53-55], we have developed an analytical model (see Sec. III in the Supplemental Material [26]) in the spirit of Ref. [34] for the scattering of EMP modes in a quantum Hall bar taking into account long range interchannel Coulomb interactions. It assumes that, in our ungated quantum Hall bars, all edge channels of the same chirality have the same velocity $v_{\mathrm{d}}(v)$ and are so strongly coupled that they see the same time-dependent potential as in Ref. [25]. Since edge states are distant for more than $1.5 \mathrm{~cm}$ from the shield of the coaxial cables located beneath the sample holder, the estimated parasitic capacitance to shield for edge states is below $1 \mathrm{fF}$ while the interedge capacitance $c_{H}$ is of the order of $0.1 \mathrm{pF}$. Therefore, Coulomb interaction effects are dominated by the interedge capacitance $C_{H}$. Finally, the dissipation of EMP modes has been neglected, a hypothesis $a$ posteriori satisfied in our samples.

In the geometry depicted in Fig. 1, the low-frequency expansion of the measured reactance is of the form

$$
\operatorname{Im}\left[Z_{23}^{(\mathrm{expt})}(\omega)\right]=i L \omega+O\left(\omega^{2}\right)
$$

where $L$ denotes the total inductance for the quantum Hall bar delimited by a dashed red box in Fig. 1(b). Because here, the magnetic inductance is much smaller than the kinetic inductance (see Supplemental Material [26], Sec. I), $L$ can be obtained from the edge-magnetoplasmon scattering model as

$$
\begin{gathered}
L=\left(R_{K} / v\right)\left[l / 2 v_{\mathrm{eff}}(v)\right], \\
v_{\mathrm{eff}}(v)=v_{\mathrm{d}}(v)\left(1+\frac{C_{q}(v)}{2 C_{H}(v)}\right) .
\end{gathered}
$$

where $l$ is the length of the Hall bar [see Fig. 1(b)], $C_{q}(v)=$ $v e^{2} l / h v_{\mathrm{d}}(v)$ is the quantum capacitance of $v$ edge channels recently measured in Corbino geometries [56], and the geometric capacitance $C_{H}(v)$ describes the effect of Coulomb interactions between counterpropagating edge channels. This is different from the quantum $R L$ circuit where, because of the gating, the capacitance $C_{H}$ has to be replaced by the capacitance $C_{g}$ with the nearby gates leading to a renormalization of $v_{\mathrm{d}}$ by $1+C_{q} / C_{g}$ for right and left moving charge density waves [24]. Here, the renormalization factor involves a $C_{H}$ capacitance with the series addition $C_{q} / 2$ of the quantum capacitances of counterpropagating edges. As a consequence, Eq. (3) still relates $L=R_{H}^{2} C_{\mu}$ to the Hall resistance and to the electrochemical capacitance [57] $C_{\mu}$ defined as the series addition of $C_{H}$ to $C_{q} / 2$. Equation (3) suggests that the inductance can be interpreted as a kinetic inductance associated with an effective time of flight $l / v_{\text {eff }}(v)$. But, as discussed in the Supplemental Material [26] (Sec. III), $v_{\text {eff }}(v)$ is neither the drift velocity for noninteracting electrons nor even a renormalized electron's velocity within chiral edge channels, but an effective velocity arising from the combination of their kinetic quantum inertia and Coulomb interactions within the quantum Hall bar. This effective inertia is of quantum origin, reflecting the minimal energy associated with an electrical current and appears, as we will see, to be dominated by the effects of Coulomb interactions.

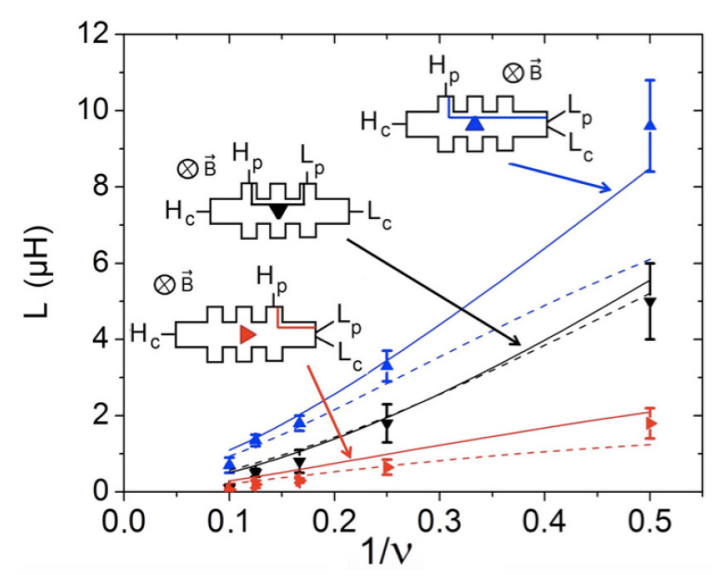

FIG. 3. For measurement configurations with $B<0$ corresponding to $l=600,1000$, and $1600 \mu \mathrm{m}$, the inductance increases with $1 / v$. Dashed lines correspond to model $v_{\mathrm{d}}(v)=v_{\mathrm{d}} / v$ with $v_{\mathrm{d}}=15$, 5 , and 40 (in units of $10^{5} \mathrm{~ms}^{-1}$ ) from top to bottom. The $v_{\mathrm{d}}(v)=$ $v_{\mathrm{d}} / \sqrt{v}$ model leads to (solid lines) $v_{\mathrm{d}}=6,3$, and 17 in units of $10^{5} \mathrm{~ms}^{-1}$ from top to bottom. The blue points corresponds to the experimental data displayed in Fig. 2.

The geometric capacitance $C_{H}(v)$ depends on the width $W$ of the sample, and of the structure and geometry of the quantum Hall edge channels (see Sec. IV of the Supplemental Material [26]), through a length $\xi_{H}(v)$ proportional to the width $W_{H}(v)$ of a single channel. Following Ref. [31], $W_{H}(v)=\left[1+\pi^{2} \alpha_{\text {eff }}(v)\right] \lambda_{H}(v)$, which is of the order of a few tens of $\mathrm{nm}$ for AlGaAs/GaAs quantum Hall systems. Finally, the interedge Coulomb interaction contribution to $v_{\text {eff }}(v)$,

$$
\frac{v_{\mathrm{d}}(\nu) C_{q}(\nu)}{2 C_{H}(\nu)} \simeq \frac{\sigma_{H}(\nu)}{2 \pi \varepsilon_{0} \varepsilon_{r}} \ln \left(\frac{W}{\nu \xi_{H}(\nu)}\right),
$$

is found to be linear in $v$, because of its proportionality to the quantum Hall conductivity $\sigma_{H}(v)$, but with a logarithmic multiplicative correction which is a signature of Coulomb interactions.

We will now discuss how this expression and the experimental data enable us to rule out some models for $v_{\mathrm{d}}(v)$. We have considered two different models for the confining potential at the edge of the sample which leads to a different prediction for $v_{\mathrm{d}}(v)$ : In Ref. [58], $v_{\mathrm{d}}(v)=\omega_{c} / k_{F}$, where $\omega_{c}=e B / m^{*}$ is the cyclotron frequency and $k_{F}=\sqrt{2 \pi / n_{S}}$ the Fermi wave vector. This leads to a dependence $v_{\mathrm{d}}(v)=v_{\mathrm{d}} / v$ whereas in Ref. [59], the gradient of the potential is proportional to $\hbar \omega_{c} / l_{B}$, where $l_{B}=\sqrt{\hbar / e B}$ is the magnetic length thereby leading to a scaling $v_{\mathrm{d}}(\nu)=v_{\mathrm{d}} / \sqrt{v}$.

Figure 3 contains the first main quantitative result of this work, i.e., the quantum inductance as a function of $1 / v$ for configurations $B<0(B>0$ configurations are analyzed in Sec. V of the Supplemental Material [26]). Values have been obtained from the reactance data depicted in Fig. 2 using the slope at low frequency of $f \mapsto X(f)$ data sets. Three configurations in which $L_{\mathrm{pot}}$ and $H_{\mathrm{pot}}$ are plugged to different contacts (see Fig. 3) and thus correspond to different $l$ have been studied. The main result is the dependence of the inductance on $1 / v$ which involves a linear part [see Eq. (3a)] due to the presence of $v$ channels in parallel, but with a nonlinear 


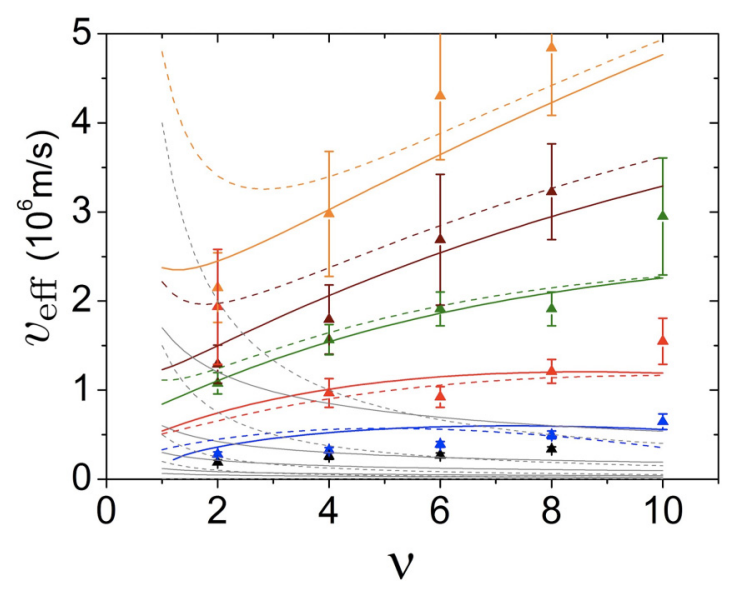

FIG. 4. $v_{\text {eff }}$ as a function of $v$. Dashed curves correspond to $v_{\mathrm{d}}(v)=v_{\mathrm{d}} / v$ and solid lines to $v_{\mathrm{d}}(v)=v_{\mathrm{d}} / \sqrt{v}$. Colored lines correspond to the expressions (1) taking into account interedge Coulomb interactions. Thin gray curves correspond to plots of the bare velocity $v_{\mathrm{d}}(v)$. The solid lines have been obtained with (starting from the top curve) $v_{\mathrm{d}}=17, v_{\mathrm{d}}=6$ (fit of the data from Fig. 2), $v_{\mathrm{d}}=3, v_{\mathrm{d}}=1.2$, and $v_{\mathrm{d}}=0.66$ in units of $10^{5} \mathrm{~ms}^{-1}$. For the dashed lines, we have starting from the top curve: $v_{\mathrm{d}}=40, v_{\mathrm{d}}=15, v_{\mathrm{d}}=5, v_{\mathrm{d}}=2$, and $v_{\mathrm{d}}=1$ in units of $10^{5} \mathrm{~ms}^{-1}$.

correction stemming from the $v$ dependence of $v_{\mathrm{d}}(v)$ [see Eqs. (3b)] together with Coulomb interaction effects [see Eq. (4)]. The different dependences of $v_{\mathrm{d}}(v)$ lead to different theoretical predictions. We find that the scaling $v_{\mathrm{d}}(v)=$ $v_{\mathrm{d}} / \sqrt{v}$ is the best for describing the experimental data.

We have then extracted the velocity $v_{\text {eff }}(v)$ using Eq. (3a) from each value of the inductance (see Fig. 4). This is the second main quantitative experimental result of this work. Each family of points corresponds to a specific sample configuration for which the sample has been heated up, rebonded, and cooled down again. These manipulations affect the electrostatic arrangement of charges at the edge, thereby modifying $v_{\mathrm{d}}$ from one experiment to the other. Figure 4 presents predictions for $v_{\text {eff }}(v)$ from Eq. (1) for different models for $v_{\mathrm{d}}(v)$. Similarly to the discussion of Fig. 3, the $v^{-1 / 2}$ scaling for $v_{\mathrm{d}}(v)$ gives the best reproduction of the experimental data. But a striking point is that in order to reproduce the experimental data, it is necessary to take into account the interchannel Coulomb interactions: Ignoring the interactions would correspond to using $v_{\mathrm{d}}(v)$ instead of $v_{\text {eff }}$ in Eq. (3a). This is shown by the thin solid and dashed gray curves in Fig. 4, which clearly do not follow the experimental data. We thus interpret the $v$ dependence of $v_{\text {eff }}(v)$ when increasing $v$ from 2 to 10 (mostly linear with a log correction) as a strong indication of the dominant role of Coulomb interactions in these ungated samples.

Let us comment on the spread of values for $v_{\mathrm{d}}$ given in Fig. 4, which reflects the variability of the electrostatic environment in the samples from one experimental cooldown to another. A variation by a factor of 25 is observed across all experiments (three higher curves for $B>0$, all others for $B<0$ ) but by only a factor of 6 when considering only one orientation of $B$. This is still much larger than the relative change of the 2DEG density but $v_{\mathrm{d}}$ reflects the edge potential, which may vary more from one experiment to the other. As we use a shallow etching technique, the sample edges are very sensitive to any change of the electric potential landscape [60]. The values that we have obtained for $v_{\mathrm{d}}(v=2)$ are compatible with estimates in the literature [61] for shallow etched samples. Moreover, our measurements of $v_{\text {eff }}(v)$ are in the same range and qualitatively show a similar $v$ dependence as the ones obtained in Ref. [62] for ungated samples by a time-of-flight technique.

To summarize, we have shown that, at low frequencies, a macroscopic quantum Hall bar is a perfect 1D conductor exhibiting a vanishing longitudinal resistance and a finite inductance. By fitting its dependence on $v$ and on the sample geometry using a simple long range effective Coulomb interaction model in the spirit of Büttiker et al. [57], we have shown that it reflects the effective quantum inertia of charge carriers within the edges of the quantum Hall bar. Contrary to the case of superconductors where carrier inertia arises from the effective mass of the Cooper pairs, here it reflects how Coulomb interactions alter the propagation of low-frequency massless edge-magnetoplasmon modes. Remarkably, the experimental data can be understood using a simple model which is formally similar to the one used for gated nanofabricated samples $[63,64]$ : Starting from chiral charge transport with bare velocity $v_{\mathrm{d}}(v)$, we include Coulomb interactions with the other edges via classical electrostatics and edge structure geometry from Ref. [31]. Going beyond this phenomenological but practical model would involve a multiscale treatment combining our approach to interchannel interactions with a self-consistent microscopic approach solving the problem of electrons in the presence of intrachannel Coulomb interactions [65-67].

Finally, macroscopic samples may provide a rescaled testbed for studying the scattering properties of edge magnetoplasmons in 1-100 $\mu \mathrm{m}$ sized samples. Studying ac transport properties of macroscopic samples up to radio frequencies could thus open the way to realizing controlled quantum linear components for quantum nanoelectronics in 1D edge channels, with possible applications to electron [68] and microwave quantum optics in ballistic quantum conductors [69].

We warmly thank G. Fève and B. Plaçais (ENS Paris) and $\mathrm{Ch}$. flindt (Aalto University) for useful discussions and suggestions, K. Ikushima (Tokyo University TUAT) for discussions at early stages of this work as well as the referees for important comments and clarifications on this manuscript. This work has been partly supported by ANR grant "1shot reloaded" (ANR-14-CE32-0017) and the French Renatech network.
[1] K. V. Klitzing, G. Dorda, and M. Pepper, Phys. Rev. Lett. 45, 494 (1980).
[2] B. I. Halperin, Phys. Rev. B 25, 2185 (1982).

[3] R. J. Haug, Semicond. Sci. Technol. 8, 131 (1993). 
[4] J. Weis and K. von Klitzing, Philos. Trans. R. Soc. A 369, 3954 (2011).

[5] M. E. Suddards, A. Baumgartner, M. Henini, and C. J. Mellor, New J. Phys. 14, 083015 (2012).

[6] T. J. Quinn, Metrologia 26, 69 (1989).

[7] B. Jeckelmann and B. Jeanneret, Rep. Prog. Phys. 64, 1603 (2001).

[8] F. J. Ahlers, B. Jeanneret, F. Overney, J. Schurr, and B. M. Wood, Metrologia 46, R1 (2009).

[9] F. Delahaye, Metrologia 31, 367 (1995).

[10] S. W. Chua, A. Hartland, and B. Kibble, IEEE Trans. Instrum. Meas. 48, 309 (1999).

[11] J. Schurr, B. Wood, and F. Overney, IEEE Trans. Instrum. Meas. 54, 512 (2005).

[12] M. E. Cage and A. Jeffrey, J. Res. Natl. Inst. Stand. Technol. 101, 733 (1996).

[13] B. Jeanneret, B. D. Hall, H.-J. Bühlmann, R. Houdré, M. Ilegems, B. Jeckelmann, and U. Feller, Phys. Rev. B 51, 9752 (1995).

[14] J. Schurr, J. Kučera, K. Pierz, and B. P. Kibble, Metrologia 48, 47 (2011).

[15] P. J. Burke, IEEE Trans. Nanotechnol. 1, 129 (2002).

[16] J. Kang, Y. Matsumoto, X. Li, J. Jiang, X. Xie, K. Kawamoto, M. Kenmoku, J. H. Chu, W. Liu, J. Mao, K. Ueno, and K. Banerjee, Nat. Electron. 1, 46 (2018).

[17] A. A. Annunziata, D. F. Santavicca, F. Frunzio, G. Catelani, M. J. Rooks, A. Frydman, and D. E. Prober, Nanotechnology 21, 445202 (2010).

[18] J. Luomahaara, V. Vesterinen, L. Grönberg, and J. Hassel, Nat. Commun. 5, 4872 (2014).

[19] G. Coiffard, K. F. Schuster, E. F. C. Driessen, S. Pignard, M. Calvo, A. Catalano, J. Goupy, and A. Monfardini, J. Low Temp. Phys. 184, 654 (2016).

[20] M. Büttiker, A. Prêtre, and H. Thomas, Phys. Rev. Lett. 70, 4114 (1993).

[21] M. Büttiker, J. Phys.: Condens. Matter 5, 9361 (1993).

[22] A. Prêtre, H. Thomas, and M. Büttiker, Phys. Rev. B 54, 8130 (1996).

[23] J. Gabelli, G. Fève, J. Berroir, B. Plaçais, A. Cavanna, B. Etienne, Y. Jin, and D. Glattli, Science 313, 499 (2006).

[24] J. Gabelli, G. Fève, T. Kontos, J.-M. Berroir, B. Plaçais, D. C. Glattli, B. Etienne, Y. Jin, and M. Büttiker, Phys. Rev. Lett. 98, 166806 (2007).

[25] L. Song, J. Yin, and S. Chen, New J. Phys. 20, 053059 (2018).

[26] See Supplemental Material at http://link.aps.org/supplemental/ 10.1103/PhysRevB.104.L121301 for detailed computations and supplementary experimental results.

[27] M. Büttiker, Phys. Rev. B 38, 9375 (1988).

[28] O. G. Balev and P. Vasilopoulos, Phys. Rev. Lett. 81, 1481 (1998).

[29] I. O. Baleva, N. Studart, and O. G. Balev, Phys. Rev. B 65, 073305 (2002).

[30] S. M. Girvin and K. Yang, Modern Condensed Matter Physics (Cambridge University Press, Cambridge, U.K., 2019).

[31] D. B. Chklovskii, B. I. Shklovskii, and L. I. Glazman, Phys. Rev. B 46, 4026 (1992).

[32] I. L. Aleiner and L. I. Glazman, Phys. Rev. Lett. 72, 2935 (1994).

[33] J. H. Han and D. J. Thouless, Phys. Rev. B 55, R1926(R) (1997).
[34] M. Hashisaka, H. Kamata, N. Kumada, K. Washio, R. Murata, K. Muraki, and T. Fujisawa, Phys. Rev. B 88, 235409 (2013).

[35] N. Hiyama, M. Hashisaka, and T. Fujisawa, Appl. Phys. Lett. 107, 143101 (2015).

[36] B. Kibble and G. Rayner, Coaxial AC bridges (Taylor \& Francis, London, 1984).

[37] K. Okada and T. Sekino, Agilent Impedance Measurement Handbook, 3rd ed. (Agilent Technologies Co. Ltd, 2003).

[38] M. Grayson and F. Fischer, J. Appl. Phys. 98, 013709 (2005).

[39] C. Hernandez, C. Consejo, P. Degiovanni, and C. Chaubet, J. Appl. Phys. 115, 123710 (2014).

[40] W. Desrat, D. K. Maude, L. B. Rigal, M. Potemski, J. C. Portal, L. Eaves, M. Henini, Z. R. Wasilewski, A. Toropov, G. Hill, and M. A. Pate, Phys. Rev. B 62, 12990 (2000).

[41] J. Melcher, J. Schurr, F. Delahaye, and A. Hartland, Phys. Rev. B 64, 127301 (2001).

[42] Here, $R_{x x}(\omega)$ denotes the frequency-dependent longitudinal dependence of the quantum Hall bar.

[43] I. Safi and H. J. Schulz, Phys. Rev. B 52, R17040(R) (1995).

[44] I. Safi, Eur. Phys. J. D 12, 451 (1999).

[45] E. Bocquillon, V. Freulon, J. Berroir, P. Degiovanni, B. Plaçais, A. Cavanna, Y. Jin, and G. Fève, Nat. Commun. 4, 1839 (2013).

[46] A. O. Slobodeniuk, I. P. Levkivskyi, and E. V. Sukhorukov, Phys. Rev. B 88, 165307 (2013).

[47] D. C. Glattli, E. Y. Andrei, G. Deville, J. Poitrenaud, and F. I. B. Williams, Phys. Rev. Lett. 54, 1710 (1985).

[48] G. Sukhodub, F. Hohls, and R. J. Haug, Phys. Rev. Lett. 93, 196801 (2004).

[49] I. Safi and H. Schulz, in Quantum Transport in Semiconductor Submicron Structures, edited by B. Kramer (Kluwer Academic, Dordrecht, 1995), p. 159.

[50] H. Steinberg, G. Barak, A. Yacobi, L. Pfeiffer, K. West, B. Halperin, and K. Le Hur, Nat. Phys. 4, 116 (2008).

[51] H. Kamata, N. Kumada, M. Hashisaka, K. Muraki, and T. Fujisawa, Nat. Nanotechnol. 9, 177 (2014).

[52] P. Brasseur, N. H. Tu, Y. Sekine, K. Muraki, M. Hashisaka, T. Fujisawa, and N. Kumada, Phys. Rev. B 96, 081101(R) (2017).

[53] P. Degiovanni, C. Grenier, G. Fève, C. Altimiras, H. le Sueur, and F. Pierre, Phys. Rev. B 81, 121302(R) (2010).

[54] C. Cabart, B. Roussel, G. Fève, and P. Degiovanni, Phys. Rev. B 98, 155302 (2018).

[55] R. Rodriguez, F. Parmentier, D. Ferraro, P. Roulleau, U. Gensser, A. Cavanna, M. Sassetti, F. Portier, D. Mailly, and P. Roche, Nat. Commun. 11, 2426 (2020).

[56] A. Delgard, B. Chenaud, D. Mailly, U. Gennser, K. Ikushima, and C. Chaubet, Phys. Status Solidi B 256, 1800548 (2019).

[57] T. Christen and M. Büttiker, Phys. Rev. B 53, 2064 (1996).

[58] S. A. Mikhailov, in Edge Excitations of Low-Dimensional Charged Systems, edited by O. Kirichek, Horizons in World Physics Vol. 236 (Nova Science, New York, 2000), Chap. 7, pp. 171-198.

[59] C. Chaubet and F. Geniet, Phys. Rev. B 58, 13015 (1998).

[60] Additional results obtained on samples from different wafers are presented in Sec. V of the Supplemental Material [26] and provide additional evidence of the robustness of our analysis.

[61] P. Roulleau, F. Portier, P. Roche, A. Cavanna, G. Faini, U. Gennser, and D. Mailly, Phys. Rev. Lett. 100, 126802 (2008).

[62] N. Kumada, H. Kamata, and T. Fujisawa, Phys. Rev. B 84, 045314 (2011). 
[63] M. Hashisaka, K. Washio, H. Kamata, K. Muraki, and T. Fujisawa, Phys. Rev. B 85, 155424 (2012).

[64] M. Hashisaka and T. Fujisawa, Rev. Phys. 3, 32 (2018).

[65] P. Armagnat, A. Lacerda-Santos, B. Rossignol, C. Groth, and X. Waintal, SciPost Phys. 7, 31 (2019).

[66] P. Armagnat and X. Waintal, J. Phys.: Mater. 3, 02LT01 (2020).

[67] G. Roussely, E. Arrighi, G. Georgiou, S. Takada, M. Schalk, M. Urdampilleta, A. Ludwig, A. D. Wieck, P. Armagnat, T. Kloss,
X. Waintal, T. Meunier, and C. Bäuerle, Nat. Commun. 9, 2811 (2018).

[68] E. Bocquillon, V. Freulon, F. Parmentier, J. Berroir, B. Plaçais, C. Wahl, J. Rech, T. Jonckheere, T. Martin, C. Grenier, D. Ferraro, P. Degiovanni, and G. Fève, Ann. Phys. (Berlin) 526, 1 (2014).

[69] A. L. Grimsmo, F. Qassemi, B. Reulet, and A. Blais, Phys. Rev. Lett. 116, 043602 (2016). 